\title{
Critical role of platelet-derived growth factor- $\alpha$ in angiogenesis after indirect bypass in a murine moyamoya disease model
}

\author{
Tomohide Hayashi, MD, PhD, ${ }^{1}$ Seiji Yamamoto, PhD, ${ }^{2}$ Takeru Hamashima, MD, ${ }^{2}$ Hisashi Mori, $\mathrm{PhD},{ }^{3}$ \\ Masakiyo Sasahara, MD, PhD, ${ }^{2}$ and Satoshi Kuroda, MD, PhD, IFAANS ${ }^{1}$ \\ Departments of ${ }^{1}$ Neurosurgery, ${ }^{2}$ Pathology, and ${ }^{3}$ Molecular Neuroscience, Graduate School of Medicine and Pharmaceutical \\ Sciences, University of Toyama, Japan
}

\begin{abstract}
OBJECTIVE This study aimed to clarify the underlying mechanism of pathognomonic angiogenesis between the temporal muscle and neocortex after indirect bypass for moyamoya disease by shedding light on the role of platelet-derived growth factor receptor- $\alpha$ (PDGFR $\alpha$ ) in angiogenesis.

METHODS The gene for PDGFR $\alpha$ was systemically inactivated in adult mice ( $\alpha$-KO mice). The Pdgfra-preserving mice (Flox mice) and $\alpha-\mathrm{KO}$ mice were exposed to bilateral common carotid artery stenosis (BCAS) by using microcoils. One week later the animals underwent encephalomyosynangiosis (EMS) on the right side. Cerebral blood flow (CBF) was serially measured using a laser Doppler flowmeter. Histological analysis was performed on the distribution of CD31-positive vessels and collagen deposit at 28 days after BCAS. Reverse transcription polymerase chain reaction (RT-PCR) was performed to assess the expression of collagen mRNA in the skin fibroblasts derived from Flox and $\alpha-\mathrm{KO}$ mice.
\end{abstract}

RESULTS BCAS significantly reduced CBF up to approximately $70 \%$ of the control level at 28 days after the onset. There was no significant difference in CBF between Flox and $\alpha-K O$ mice. EMS significantly enhanced the improvement of CBF on the ipsilateral side of Flox mice, but not $\alpha$-KO mice. EMS significantly induced the development of CD31-positive vessels in both the neocortex and temporal muscle on the ipsilateral side of Flox mice, but not $\alpha-K O$ mice. Deposition of collagen was distinctly observed between them in Flox mice, but not $\alpha-\mathrm{KO}$ mice. Expression of mRNA of collagen type 1 alpha 1 (Col1a1) and collagen type 3 alpha 1 (Co/3a1) was significantly downregulated in the skin fibroblasts from $\alpha-K O$ mice.

CONCLUSIONS This is the first study that denotes the role of a specific growth factor in angiogenesis after EMS for moyamoya disease by inactivating its gene in mice. The findings strongly suggest that PDGFR $\alpha$ signal may play an important role in developing spontaneous angiogenesis between the temporal muscle and neocortex after EMS in moyamoya disease.

https://thejns.org/doi/abs/10.3171/2020.3.JNS193273

KEYWORDS moyamoya disease; platelet-derived growth factor- $\alpha$; indirect bypass; chronic cerebral ischemia; angiogenesis; vascular disorders

$\mathrm{M}$ OYAMOYa disease is an uncommon cerebrovascular disease characterized by progressive stenosis of terminal segments of the internal carotid artery (ICA) and its main branches within the circle of Willis. The stenotic lesion leads to the marked dilatation of perforating arteries (moyamoya vessels)., ${ }^{1,2}$ Clinical symptoms of moyamoya disease include ischemic attacks such as transient ischemic attack and ischemic stroke, whereas a certain subgroup of adult patients develop intracranial bleeding. Recent studies have shown that surgical revascularization can resolve hemodynamic impairment and reduce the risk of subsequent ischemic/hemorrhagic stroke. Of these treatments, direct bypass procedures such as superficial temporal artery-middle cerebral artery anastomosis can immediately increase cerebral blood flow $(\mathrm{CBF})$ after surgery, although the procedure is sometimes

ABBREVIATIONS BCAS = bilateral common carotid artery stenosis; $\mathrm{CBF}=$ cerebral blood flow; $C C A=$ common carotid artery; $E C M=$ extracellular matrix; EMS $=$ encephalomyosynangiosis; ICA = internal carotid artery; $\mathrm{PCR}=$ polymerase chain reaction; $\mathrm{PDGF}$ = platelet-derived growth factor; $\mathrm{PDGFR}=\mathrm{PDGF}$ receptor; $\mathrm{RT}-\mathrm{PCR}=$ reverse transcription PCR.

SUBMITTED December 4, 2019. ACCEPTED March 16, 2020.

INCLUDE WHEN CITING Published online May 22, 2020; DOI: 10.3171/2020.3.JNS193273. 
challenging. ${ }^{3}$ On the other hand, indirect bypass is very specific for moyamoya disease. Indirect bypass surgery readily stimulates spontaneous angiogenesis between the brain surface and the vascularized donor tissues, including the temporal muscle. The procedures are technically simple to do and have been widely used, although the precise mechanisms of spontaneous angiogenesis through indirect bypass are still obscure. ${ }^{1}$

Previously, several investigators have conducted animal experiments to simulate angiogenesis after indirect bypass surgery, using several species. ${ }^{4-8}$ Chronic cerebral ischemia was produced by ligating the bilateral carotid arteries of rats, and subsequently the temporal muscle was attached onto the brain surface. Based on the results, the connective tissue formation between them was considered quite important to induce angiogenesis. ${ }^{79}$ However, the underlying mechanisms of angiogenesis after indirect bypass surgery are not understood.

The family of platelet-derived growth factors (PDGFs) includes PDGF-A, PDGF-B, PDGF-C, and PDGF-D. Two types of PDGF receptors (PDGFRs), namely PDGFR $\alpha$ and PDGFR $\beta$, undertake the overlapping but distinctive signal transduction in the various kinds of cells. ${ }^{10}$ In the CNS, PDGF-A is known to promote the proliferation of oligodendrocyte progenitor cells that express PDGFR $\alpha .{ }^{11}$ Increased PDGFR $\alpha$ signaling leads to the hyperplasia of connective tissue and an increased deposition of extracellular matrix (ECM). ${ }^{12}$ Thus, PDGFR $\alpha$ may be one of the relevant targets to regulate the remodeling of connective tissue. Indeed, the upregulation of PDGF-A and PDGFR $\alpha$ improves wound healing of skin. ${ }^{12}$ Knockout of the PDGFR $\alpha$ gene reduces the newly vascularized areas and suppresses the deposition of collagen in a process of connective tissue remodeling. ${ }^{13}$

Based on these considerations, the present study aimed to evaluate the roles of PDGFR $\alpha$ in spontaneous angiogenesis after indirect bypass surgery in moyamoya disease. For this purpose, the murine model of chronic cerebral ischemia was first used to simulate indirect bypass for moyamoya disease.

\section{Methods}

\section{Experimental Animals}

All experimental animal procedures were approved by the Committee for Institutional Animal Care and Use at the University of Toyama. Eight-week-old male C57BL/6J mice (CLEA Japan Co., Ltd.) were used. All mice $(\mathrm{N}=$ 37) were housed at $25^{\circ} \mathrm{C}$ with a 12 -hour light/dark cycle and with free access to pellet chow and water.

\section{PDGFR $\alpha$ Knockout Mice}

Mutant mice harboring a genetically mutated Pdgfra gene in which exons 4 to 5 of Pdgfra were flanked by two loxP sequences ( $\left.P d g f r a^{f l o x} / f o x\right)$ were generated as reported before. ${ }^{13}$ A bacterial artificial chromosome genomic clone (RP24-148N) originating from the DNA of C57BL/6J mice and containing Pdgfra was obtained from the BACPAC Resource Center CHORI. The targeting vector included a DNA fragment containing a loxP sequence and $p g k-n e o$ cassette flanked by two Flp recogni- tion target $(f r t)$ sites inserted into pMC1DTABGHA with bovine growth hormone-derived polyadenylation signal. The embryonic stem cell line RENKA, derived from the C57BL/6N strain, was used and the targeting vector was electroporated as previously described.

The male chimeric mice thus obtained were crossed with female CAG-PLPe deletion mutant mice to remove the $p g k-n e o$ selection cassette and obtain the heterozygous $P d g f r a^{f o x /+}$ strain. Genotyping was performed by polymerase chain reaction (PCR) using the following primers: forward, 5'-ATGCCAAACTCTGCCTGATTGA-3' and reverse, 5'-CTCACGGAACCCCCACAAC-3'. The mice of the heterozygous $P d g f r a^{f l o x /+}$ strain were cross-bred with chicken $\beta$-actin-promoter/CMV-enhancer-driven Cre-transgenic mice harboring tamoxifen-inducible Cre recombinase $\left(\mathrm{Cre}-\mathrm{ER}^{+-}\right.$; CAGGCre-ER ${ }^{+/-}$; Jackson Laboratories). The resulting offspring were mice harboring CAGGCre-ER ${ }^{+-} ; \quad P d g$ fra ${ }^{\text {flox/flox }}$ (CreER-Flox mice) or Pdgfrafloxffox (Flox mice). Mice with systemic Pdgfra inactivation (i.e., PDGFR $\alpha$ knockout $[\alpha-K O]$ mice) were obtained by tamoxifen administration $(225 \mathrm{mg} / 1 \mathrm{~kg}$ body weight, 5 consecutive days; Sigma-Aldrich) to CreER-Flox mice at 7 weeks of age. Flox mice were treated identically and used as controls.

\section{Experiment Protocol}

In this study the experiments were divided into two sessions. In the first session, the Flox $(n=10)$ and $\alpha-K O$ mice $(n=12)$ underwent bilateral common carotid artery stenosis (BCAS) with 0.16-mm-diameter microcoils. Subsequently, $\mathrm{CBF}$ was serially monitored through 4 weeks after the onset of BCAS. Sham-operated C57BL/6J mice were prepared as the control $(\mathrm{n}=5)$.

In the second session, encephalomyosynangiosis (EMS) was achieved on the right side at 7 days after the onset of BCAS. The effects of EMS on CBF and angiogenesis were precisely evaluated in the Flox and $\alpha-\mathrm{KO}$ mice $(\mathrm{n}=5$ in each group).

\section{Bilateral Common Carotid Artery Stenosis}

To produce a murine model of chronic cerebral ischemia, the mice were anesthetized with $2.0 \%$ sevoflurane. All surgical procedures were performed under a surgical microscope. Their core temperature was kept at $36.5^{\circ} \mathrm{C}-$ $37.5^{\circ} \mathrm{C}$ throughout the procedures. The bilateral common carotid arteries (CCAs) were dissected through a midline cervical incision. Two 4-0 silk sutures were placed between the CCA and the vagal nerve. While the artery was lifted by these sutures, Microcoils with an inner diameter of $0.16 \mathrm{~mm}$ (Sawane Spring Co., Ltd.) were applied to the CCAs on both sides to narrow the lumen (Fig. 1A). ${ }^{9}$

\section{CBF Measurement}

The CBF was recorded at $1 \mathrm{~mm}$ lateral and $2.5 \mathrm{~mm}$ caudal to the bregma over the skull on both sides by using a laser Doppler flowmeter (ALF21; Advance Co., Ltd.) before the onset of BCAS. The CBF measurement was repeated just after the onset of BCAS, at 7 days, and at 28 days post-BCAS. 


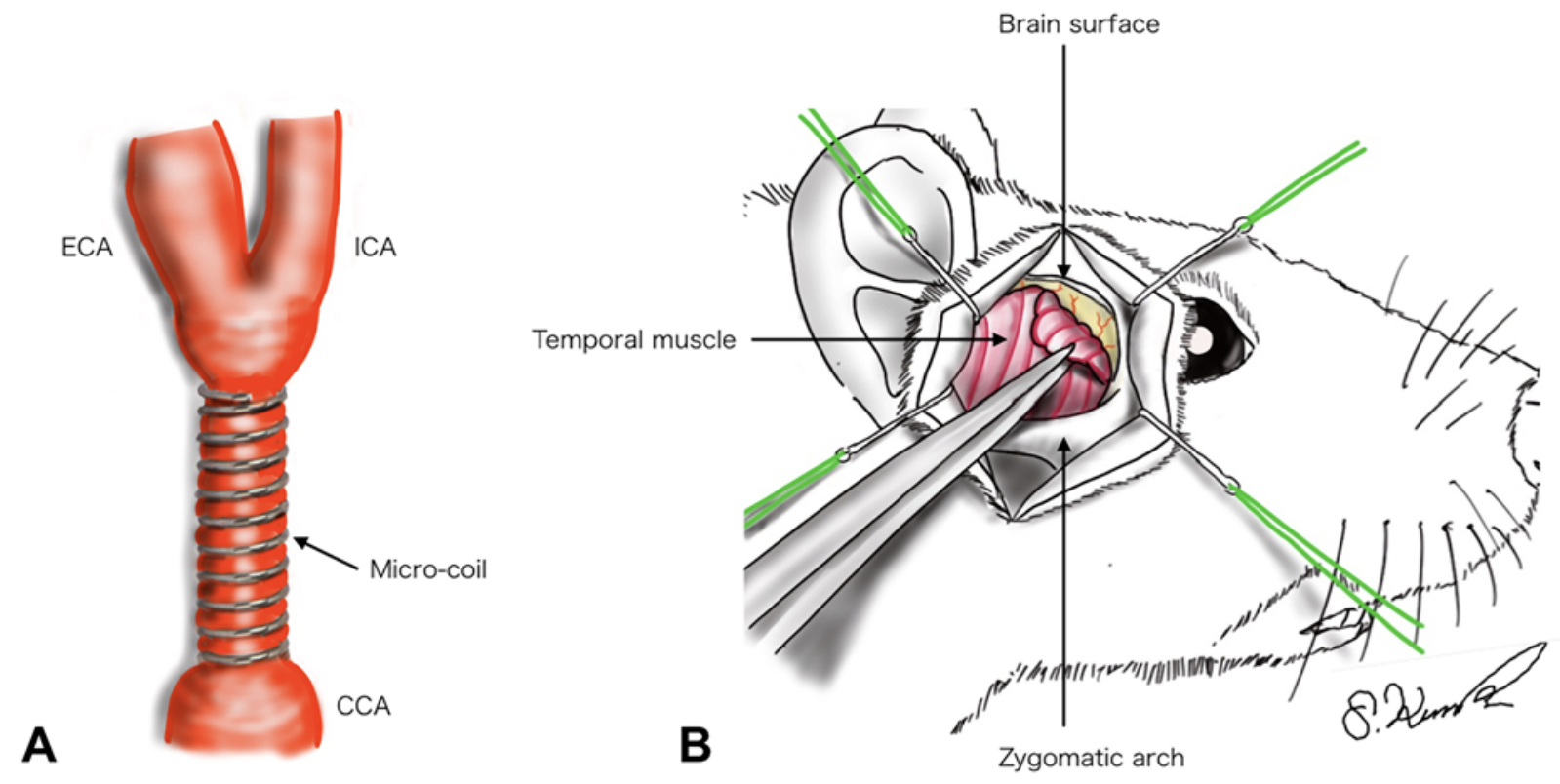

FIG. 1. Digital illustrations presenting the experiment procedures. A: The microcoils with an inner diameter of $0.16 \mathrm{~mm}$ were applied to the CCAs on both sides. B: EMS was performed by attaching the temporal muscle onto the brain surface through a small craniectomy. ECA = external carotid artery. Figure is available in color online only.

\section{Encephalomyosynangiosis}

EMS was achieved on the right side at 7 days postBCAS, as reported before., ${ }^{4,5}$ Briefly, the temporal muscle was exposed and scraped from the temporal bone on the right side. Then, a craniectomy was performed with a diamond-tip drill. The craniectomy was extended from the superior temporal line to the level of the zygomatic arch, and also from the coronal suture to the lambdoid suture. The temporal bone and dura mater were carefully removed, and the temporal muscle was attached onto the brain surface (Fig. 1B). In addition to Flox and $\alpha-\mathrm{KO}$ mice $(n=5$ in each group), Flox mice that were not subjected to BCAS also underwent EMS on the right side as the control $(n=5)$. The value of CBF was expressed as the right-to-left ratio to evaluate the effect of EMS on the right hemisphere.

\section{Histological Analysis}

The mice were deeply anesthetized with sodium pentobarbital at 28 days post-BCAS and were perfused with phosphate-buffered saline ( $\mathrm{pH}$ 7.4) through the left ventricle, followed by $4 \%$ paraformaldehyde. The brain tissue was removed together with the temporal muscle and was fixed with $4 \%$ paraformaldehyde overnight at $4^{\circ} \mathrm{C}$. The tissues were embedded in paraffin and were sliced into 3 - $\mu$ m-thick coronal sections.

For immunohistochemistry, the sections were deparaffinized and heated for 2 minutes by a pressure pot to restore antigenicity. The primary antibody against CD31 (diluted 1:50, Abcam 28364) was applied overnight at $4^{\circ} \mathrm{C}$. The sections were incubated with a biotinylated secondary antibody for 30 minutes at room temperature. Then, the sections were stained with diaminobenzidine and counterstained with hematoxylin. The number and diameter of CD31-positive blood vessels in the neocortex adjacent to the temporal muscle were quantified in 10 randomly selected fields $(400 \mu \mathrm{m} \times 400 \mu \mathrm{m}$ per field). They were also evaluated in the contralateral neocortex. The percentage of the area surrounded by CD31-positive cells in the temporal muscle was also quantified at the site of EMS. All images were photographed with an All-in-One Microscope (BZ9000; Keyence Co.) at a magnification of $\times 200$. The number and diameter of blood vessels were analyzed with image software (BZ-II analyzer; Keyence Co.).

Sirius red staining was used to observe collagen deposition at the area where EMS was performed. The percentage of the collagen-deposited area in the temporal muscle attached onto the brain was quantified as described above, using image analysis software (ImageJ 1.48v; National Institutes of Health). 27,28

\section{Reverse Transcription PCR}

Using reverse transcription (RT) PCR, the expression of the following mRNA was precisely compared between the skin fibroblasts of Flox mice and those of $\alpha$-KO mice: collagen type 1 alpha 1 (Collal) and collagen type 3 alpha 1 (Col3al). Total RNA was isolated from the skin fibroblast using the RNeasy Mini Kit (Qiagen). RT-PCR was conducted with a Thermal Cycler Dice Real System TP800 (Takara). The cDNAs were diluted 1:25 in the reaction mixture consisting of SYBR Premix EX taq II (Takara). The RT-PCR program consisted of hot-start enzyme activation at $95^{\circ} \mathrm{C}$ for 10 seconds, 40 cycles of amplification at $95^{\circ} \mathrm{C}$ for 10 seconds, and then $60^{\circ} \mathrm{C}$ for 40 seconds. Finally, to obtain the dissociation curve, a final cycle was performed at $95^{\circ} \mathrm{C}$ for 1 minute, $60^{\circ} \mathrm{C}$ for 30 seconds, and then $95^{\circ} \mathrm{C}$ for 10 seconds. For data analysis, mouse $\beta$-actin $(A c t b)$ was used as an internal control. Induction values 

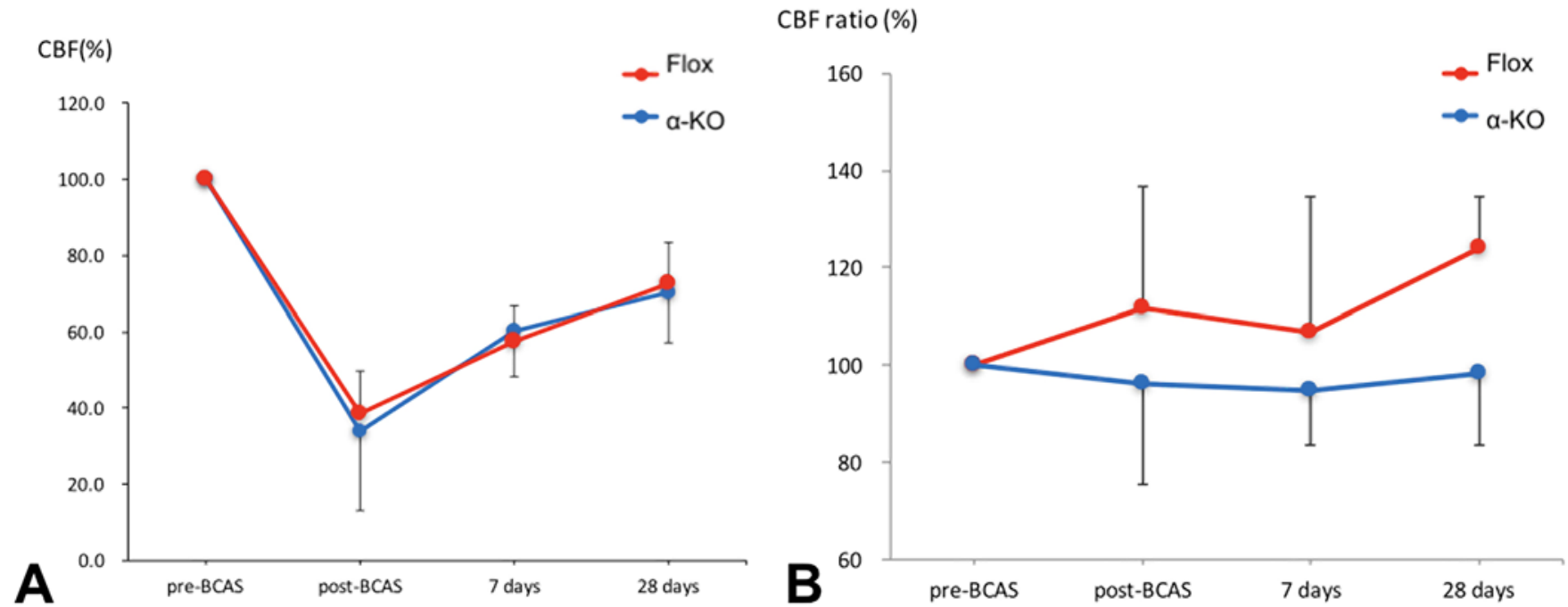

FIG. 2. Line graphs showing serial CBF change during 28 days after BCAS in Flox and $\alpha-K O$ mice. Note that there is no significant difference between the two groups. A: Line graph showing a serial change in the ratio of the EMS-side CBF to the non-EMS-side CBF. EMS significantly improved CBF on the EMS side of Flox mice at 28 days after the onset of BCAS, but CBF did not differ from the non-EMS side even after EMS in $\alpha-K O$ mice. B: There was a significant difference in the CBF ratios between Flox and $\alpha-K O$ mice at 28 days after BCAS $(p<0.05)$. Figure is available in color online only.

were calculated using soft analysis software. The primer sequence is available upon request from the Takara Bio, Inc., website (http://www.takara-bio.co.jp/).14,29

\section{Statistical Analysis}

Data were expressed as mean $\pm \mathrm{SD}$. Continuous variables were compared using a 2-tailed unpaired t-test between two groups or a 1-factor ANOVA among three groups, followed by the Tukey-Kramer method as appropriate. Categorical variables were compared using a chisquare test. Differences were judged to be statistically significant when the $\mathrm{p}$ value was $<0.05$.

\section{Results \\ CBF Change After BCAS}

There were no deaths after the onset of BCAS. As shown in Fig. 2A, CBF markedly decreased to $38.4 \% \pm$ $11.4 \%$ and $34.2 \% \pm 21.0 \%$ of the control values just after BCAS in Flox $(n=10)$ and $\alpha-K O$ mice $(n=12)$, respectively ( $\mathrm{p}=0.5494)$. Then, $\mathrm{CBF}$ spontaneously improved to $57.6 \% \pm 9.4 \%$ and $60.4 \% \pm 12.0 \%$ at 7 days post-BCAS in Flox and $\alpha-\mathrm{KO}$ mice, respectively $(\mathrm{p}=0.5501)$. CBF further improved to $72.7 \% \pm 10.8 \%$ and $70.2 \% \pm 13.1 \%$ at 28 days post-BCAS in Flox and $\alpha-\mathrm{KO}$ mice, respectively ( $\mathrm{p}$ $=0.6279$ ). Therefore, the density of cerebral ischemia did not differ between them throughout the experiment.

\section{Effect of EMS on Ipsilateral CBF}

Figure 2B demonstrates serial changes of the right-toleft $\mathrm{CBF}$ ratio after the onset of BCAS. The values just after the onset of BCAS were $111.6 \% \pm 25.1 \%$ and $96.1 \%$ $\pm 20.6 \%$ in Flox and $\alpha-\mathrm{KO}$ mice, respectively. The values were $106.7 \% \pm 27.7 \%$ and $94.7 \% \pm 11.2 \%$ in Flox and
$\alpha-\mathrm{KO}$ mice at 7 days post-BCAS, respectively. Therefore, the degree of cerebral ischemia was almost equal between the hemispheres in the two groups during 7 days postBCAS. Then, they underwent EMS on the right side.

$\mathrm{CBF}$ measurement was repeated at 28 days post-BCAS, i.e., at 21 days after EMS. Based on these measurements, the right-to-left $\mathrm{CBF}$ ratio was $123.2 \% \pm 13.1 \%$ in Flox mice, being significantly higher than the $98.5 \% \pm 14.8 \%$ measured in $\alpha-\mathrm{KO}$ mice $(\mathrm{p}=0.0147)$. Therefore, EMS significantly improved ipsilateral CBF in Flox mice but not in $\alpha-\mathrm{KO}$ mice.

\section{The Effects of EMS on Angiogenesis Through Temporal Muscle}

Immunostaining against CD31 was performed to elucidate the mechanism through which EMS improved CBF after BCAS in the Flox mice but not in the $\alpha-\mathrm{KO}$ mice. Figure 3 shows representative findings in the neocortex adjacent to the temporal muscle at 21 days after EMS. Flox mice had a tight contact between the temporal muscle and neocortex, but $\alpha-\mathrm{KO}$ mice had a significant gap between them. Quantitative measurements demonstrated that EMS significantly increased the diameter of ipsilateral CD31positive vessels $(\mathrm{p}=0.0005$; Fig. $4 \mathrm{~A})$ and the number of CD31-positive vessels in the neocortex $(p=0.0109$; Fig. $4 \mathrm{~B}$ ) in the Flox mice subjected to BCAS, when compared with vessels in the contralateral side. However, these findings were not observed in $\alpha-\mathrm{KO}$ mice or the control Flox mice without BCAS.

Figure 3 also demonstrates that EMS significantly increased the area surrounded by CD31-positive cells in the temporal muscle in the Flox mice subjected to BCAS, compared with $\alpha-\mathrm{KO}$ mice as well as the control Flox mice without BCAS. This measurement showed a statistically significant difference among the three groups $(\mathrm{p}=$ 


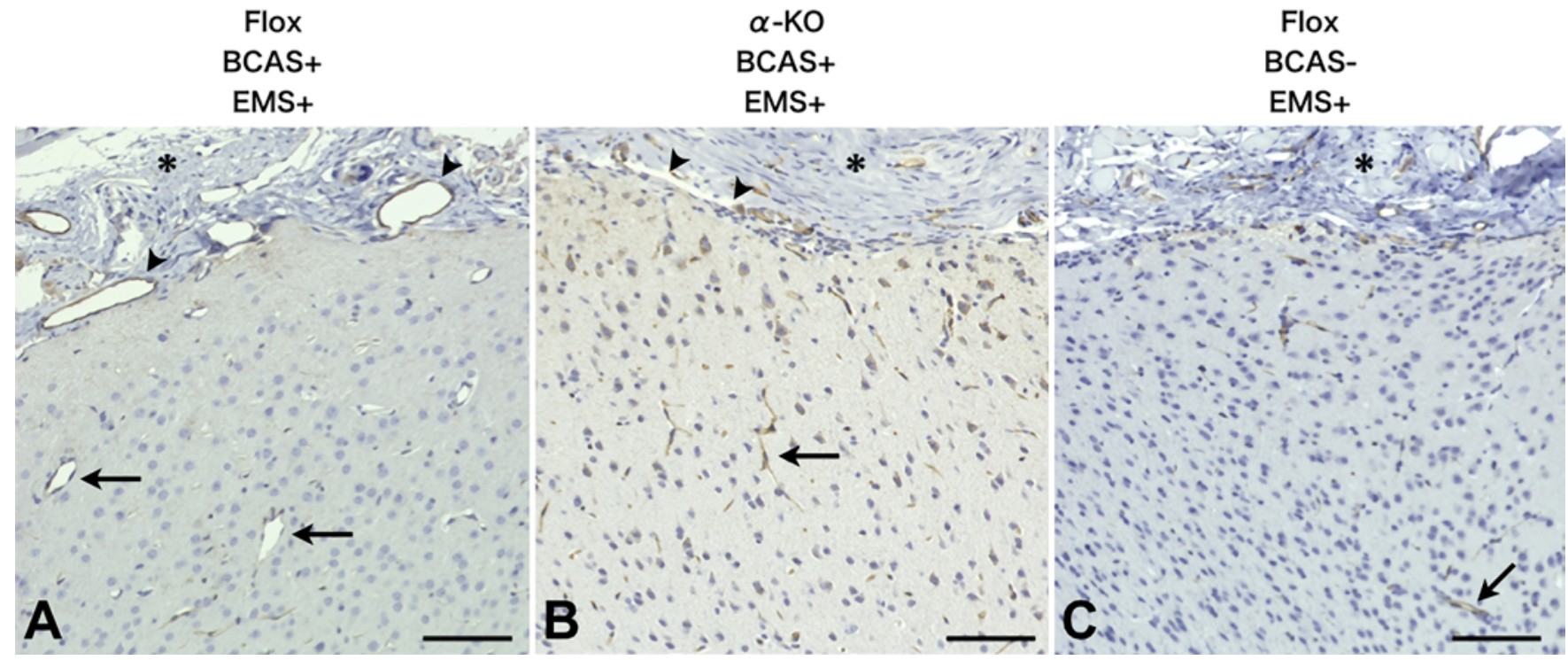

FIG. 3. Photomicrographs of immunohistochemistry against CD31 in the temporal muscle (upper portion of panels) and neocortex (lower portion of panels) after EMS in the Flox mice subjected to BCAS (A), the $\alpha-K O$ mice subjected to BCAS (B), and the control Flox mice without BCAS (C). Arrows indicate CD31-positive blood vessels in the neocortex. Asterisks indicate muscle tissue attached to cerebral cortex after EMS. Arrowheads in panel A indicate dilated blood vessels, whereas arrowheads in panel B indicate a gap between the neocortex and attached temporal muscle after EMS. Bar $=100 \mu \mathrm{m}$. Figure is available in color online only.

0.0039; Fig. 4C), with the highest area in Flox mice with BCAS among these three groups of mice.

\section{EMS-Induced Accumulation of Collagen}

Sirius red staining was performed to clarify whether or not angiogenesis between the temporal muscle and cortex depended on collagen accumulation after EMS. As shown in Fig. 5, the collagen fibers between the temporal muscle and neocortex were very dense and very tightly adhered to the surface of neocortex in the Flox mice subjected to BCAS. On the other hand, the formation of the collagen network was sparse, and the adhesion to the surface of neocortex was minimal in the $\alpha-\mathrm{KO}$ mice.

The collagen-positive area was $28.9 \% \pm 4.5 \%$ in the Flox mice subjected to BCAS, being significantly higher than $21.6 \% \pm 4.1 \%$ in $\alpha-\mathrm{KO}$ mice subjected to BCAS and $15.8 \% \pm 2.8 \%$ in the control Flox mice without BCAS (p $=0.0015)$.

\section{RT-PCR}

The expression of both Collal and Col3al mRNA was significantly downregulated in the skin fibroblasts derived from $\alpha-\mathrm{KO}$ mice and in those from Flox mice $(\mathrm{p}=0.0390$ and $\mathrm{p}<0.0001$, respectively), which supports the histological findings (Fig. 6).

\section{Discussion}

As mentioned above, previous studies were conducted to simulate indirect bypass surgery for moyamoya disease, using a rat model of bilateral CCA occlusion. ${ }^{4-8}$ Thus, $\mathrm{Ku}-$ saka et al. first used this model for their experiment and performed EMS surgery at 7 days after the onset of bilateral CCA occlusion. They found that the injection of vascular endothelial growth factor (VEGF) plasmid into the temporal muscle enhanced angiogenesis between the temporal muscle and brain. ${ }^{15}$ From the same laboratory, Hiramatsu et al. also reported that combined gene therapy (VEGF + apelin) further enhanced angiogenesis in this model. ${ }^{6}$ In addition, Kim et al. demonstrated a similar effect of bone marrow stromal cell injection in the same model..$^{16}$ Therefore, a rat bilateral CCA occlusion model is considered acceptable to simulate the effect of EMS on moyamoya disease, although it remains to be seen whether the rat bilateral CCA occlusion model completely simulates the pathogenesis and pathophysiology in moyamoya disease.

In the case of mice, however, this procedure is known to cause a high rate of mortality, probably because of more dense cerebral ischemia. ${ }^{17}$ In fact, Vajkoczy's group has used the murine model of right-sided CCA occlusion and performed EMS on the ipsilateral side. ${ }^{5,18}$ However, it is impossible to evaluate serial CBF changes by using the contralateral side as the non-EMS control in the unilateral CCA occlusion model. In this study, therefore, we applied the microcoils to the bilateral CCAs of mice to produce stenosis. As the results show, the BCAS technique could constantly induce cerebral ischemia throughout the 4 weeks after onset, which enabled us to use a genetically modified murine model of chronic cerebral ischemia and to directly assess the role of PDGFR $\alpha$ on EMS-induced angiogenesis.

As described above, EMS is known as one of the indirect bypass procedures for moyamoya disease and has been widely performed all over the world. ${ }^{1}$ In this study, EMS significantly improved CBF on the ipsilateral side but not on the contralateral side in the Flox mice, which simulated surgical results in patients with moyamoya dis- 

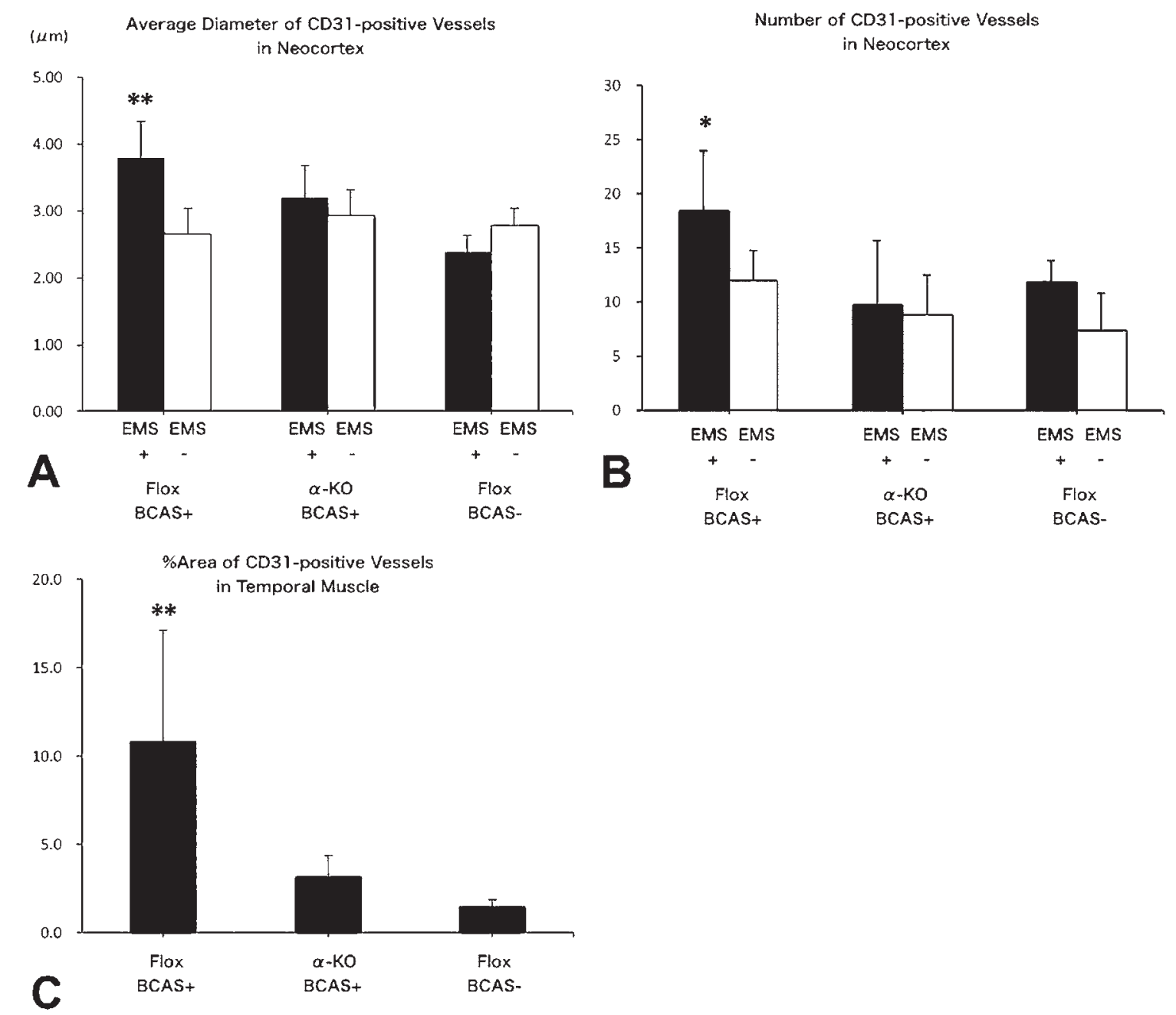

FIG. 4. Bar graphs demonstrating the quantitative data on immunohistochemistry against CD31. A: The diameter of CD31-positive vessels in the neocortex was significantly larger on the EMS side than on the non-EMS side in the Flox mice subjected to BCAS $\left({ }^{* *} p<0.01\right)$. The difference between them was not observed in $\alpha-K O$ mice subjected to BCAS and the control Flox mice without BCAS. B: The number of CD31-positive vessels in the neocortex was significantly larger on the EMS side than on the non-EMS side in the Flox mice subjected to BCAS ( $\left.{ }^{*} p<0.05\right)$. The difference between them was not observed in $\alpha-K O$ mice subjected to BCAS and the control Flox mice without BCAS. C: The percentage of vessel areas surrounded by CD31-positive endothelial cells in the temporal muscle was significantly larger in Flox mice subjected to BCAS than in $\alpha-\mathrm{KO}$ mice subjected to BCAS and control Flox mice without BCAS $\left(^{* *} p<0.01\right)$.

ease very well. Laser Doppler flowmetry is known to be very useful to continuously monitor the relative changes in CBF. Previous reports have also shown that laser Doppler flowmetry is a reliable modality to intermittently monitor CBF during several weeks in animal experiments, although investigators should be very careful when positioning the probe. ${ }^{19}$ In the present study, therefore, we evaluated the effect of EMS on CBF by using the right-to-left ratio of $\mathrm{CBF}$ but not the absolute value of $\mathrm{CBF}$.

In this study the timing of histological examinations was set at 21 days after EMS for the following reasons. First, a majority of previous studies confirmed the development of angiogenesis between the temporal muscle and brain at 14 days after EMS in the rats and mice..$^{5,6,15}$ More importantly, Marushima et al. performed EMS in the mice subjected to unilateral CCA occlusion and found significant improvement of cerebral vascular reserve at 21 days after EMS. ${ }^{18}$
In our findings, immunohistochemistry against CD31 strongly supported the results of laser Doppler flowmetry. Thus, EMS significantly developed CD31-positive vessels in both the neocortex and temporal muscle on the EMS side, but not on the non-EMS side. Interestingly, such postEMS angiogenesis was not observed in the control Flox mice without BCAS. These findings strongly suggest that EMS-induced angiogenesis occurred in response to chronic cerebral ischemia and contributed to improvement in $\mathrm{CBF}$ on the EMS side. However, these therapeutic effects were cancelled in the $\alpha-\mathrm{KO}$ mice subjected to BCAS. In addition, EMS-induced angiogenesis did not occur in their temporal muscle and neocortex. These findings strongly indicate the critical role of PDGFR $\alpha$ in angiogenesis between the temporal muscle and neocortex after EMS.

This study also demonstrated that BCAS per se significantly enhanced the deposition of collagen between 

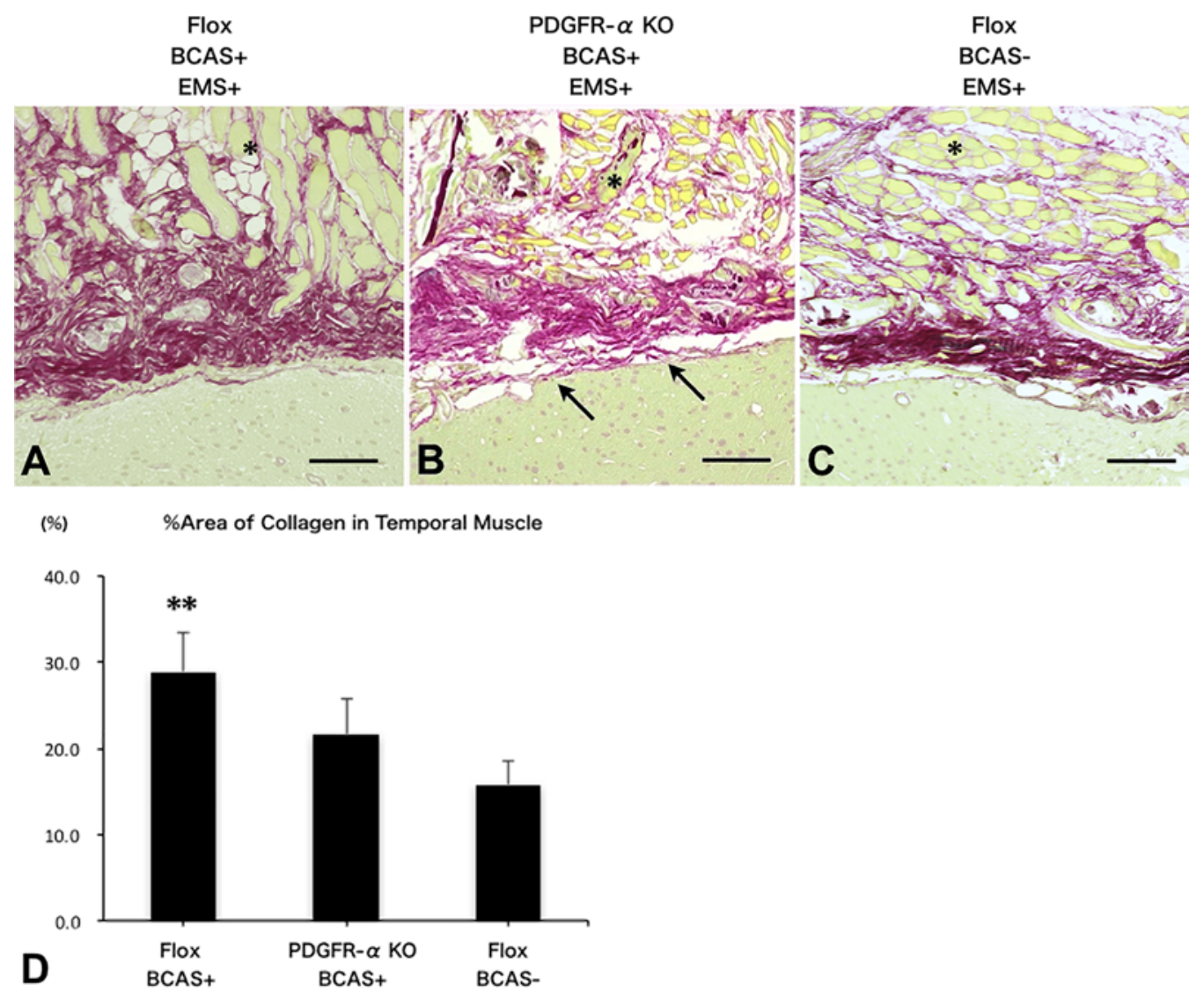

FIG. 5. Photomicrographs of Sirius red staining in the temporal muscle (upper portion of panels, asterisks) and neocortex (lower portion of panels) after EMS in the Flox mice subjected to BCAS (A), the $\alpha-K O$ mice subjected to BCAS (B), and the control Flox mice without BCAS (C). Note that there are significant gaps between the temporal muscle and brain in the $\alpha-K O$ mice (B, arrows). Bar $=100 \mu \mathrm{m}$. The bar graph (D) shows that the percentage of Sirius red-positive area of collagen in the temporal muscle was significantly larger in the Flox mice subjected to BCAS than in $\alpha-K O$ mice subjected to BCAS and the control Flox mice without BCAS $\left({ }^{* *} p<0.01\right)$. Figure is available in color online only.

the temporal muscle and neocortex in the Flox mice, suggesting that cerebral ischemia augments the formation of a collagen network between them after EMS. However, the knockout of PDGFR $\alpha$ significantly inhibited the degree of collagen deposition between the temporal muscle and neocortex. Therefore, inactivation of the Pdgfra gene significantly suppressed all phenomena occurring after EMS in the Flox mice subjected to BCAS, including the collagen deposition and angiogenesis between the temporal muscle and neocortex, and finally CBF improvement. Furthermore, RT-PCR analysis clearly showed that inactivation of the Pdgfra gene significantly downregulated the expression of Collal and Col3al mRNA. In the human body, most of the collagen is type 1 collagen, and type 3 collagen is usually found alongside type 1 collagen. ${ }^{13}$ Therefore, the deposition of collagen may greatly contribute to the initiation of spontaneous angiogenesis after EMS. In fact, recent studies have shown that collagen is an essential ECM to induce angiogenesis during wound healing. Collagen is known to enhance angiogenesis by developing the matrix framework for new vessel sprouting,,$^{20,21}$ and to support the proliferation, survival, and migration of endothelial cells. ${ }^{22,23}$ Furthermore, Horikawa et al. recently implanted

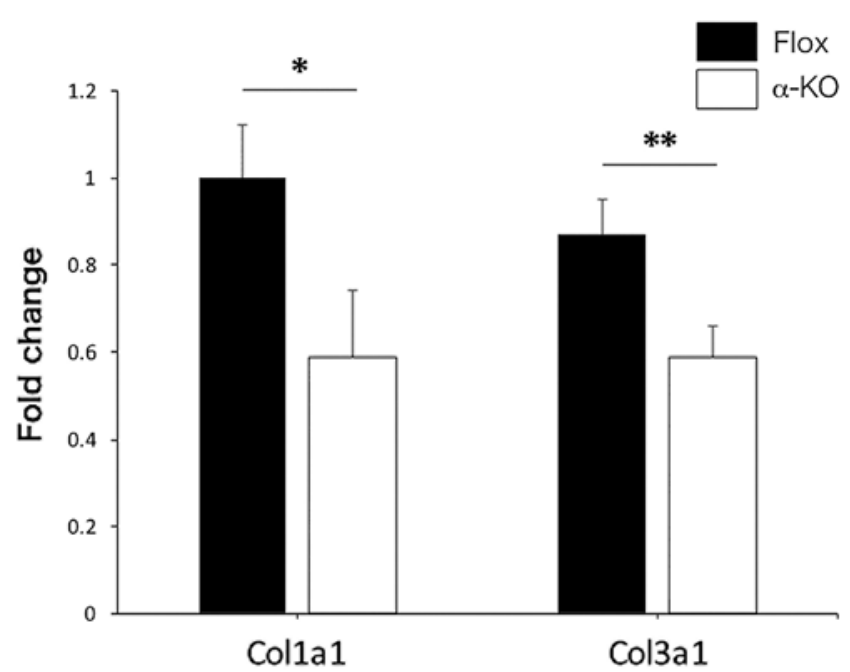

FIG. 6. Bar graph demonstrating the mRNA expression of Col1a1 and Col3a1 in the fibroblasts derived from the Flox and $\alpha-K O$ mice. ${ }^{*} p<0.05$ and ${ }^{* *} p<0.01$. 
sponge matrices into the subcutaneous space of mice and found that inactivation of the Pdgfra gene significantly inhibits both angiogenesis and ECM deposition in sponge matrices. ${ }^{13}$ Nakamura et al. also performed EMS in pigs subjected to an ICA occlusion and found active proliferation of macrophages and angiogenesis within the newly developed connective tissues between the temporal muscle and brain, which supports the present results. ${ }^{7}$ Therefore, we believe that the present findings would contribute to the development of therapeutic strategies to enhance spontaneous and/or surgical angiogenesis for patients with moyamoya in the future.

There are only a few studies that assess the role of the PDGF family in moyamoya disease. ${ }^{14}$ Aoyagi et al. cultured the smooth muscle cells derived from the scalp artery of patients with moyamoya and reported their reduced expression of PDGFR and reduced response to PDGF. ${ }^{24,25}$ Kang et al. reported that the plasma concentration of PDGF was increased in patients with moyamoya when compared with the healthy controls. ${ }^{26}$ Very recently, Marushima et al. found that transfection of the $P D G F-B B$ gene enhanced collateral vessel formation after EMS in mice. ${ }^{18}$ However, research on the role of PDGF in moyamoya disease has just begun. Therefore, further studies are warranted to evaluate the role of PDGF in pathognomonic angiogenesis after indirect bypass in moyamoya disease.

\section{Conclusions}

A murine model of chronic cerebral ischemia enabled us to evaluate the role of a specific growth factor in angiogenesis after EMS for moyamoya disease by inactivating its gene. The present findings strongly suggest that PDGFR $\alpha$ may play an essential role in developing spontaneous angiogenesis between the temporal muscle and neocortex after indirect bypass surgery in ischemic brain.

\section{Acknowledgments}

This study was partly supported by a grant from the Research Committee on Moyamoya Disease, the Japanese Ministry of Health, Labour and Welfare (Dr. Kuroda). Part of this work was also supported by Grants-in-Aid for Scientific Research Nos. JP25293093/JP17H04062 (Dr. Sasahara) and JP23122506/ JP26460360 (Dr. Yamamoto) from the Ministry of Education, Culture, Sports, Science and Technology, Japan.

We thank Yumiko Hayakawa at the Department of Neurosurgery, University of Toyama; Youichi Kurashige, Takako Matsushima, and Miwa Fujikawa at the Department of Pathology, Graduate School of Medicine and Pharmaceutical Sciences, University of Toyama, Japan; and Toshihiko Fujimori at the Division of Embryology, National Institute for Basic Biology, Okazaki, Japan, for their technical assistance.

\section{References}

1. Kuroda S, Houkin K. Moyamoya disease: current concepts and future perspectives. Lancet Neurol. 2008;7(11):10561066.

2. Suzuki J, Takaku A. Cerebrovascular "moyamoya" disease. Disease showing abnormal net-like vessels in base of brain. Arch Neurol. 1969;20(3):288-299.

3. Arikan F, Vilalta J, Torne R, et al. Rapid resolution of brain ischemic hypoxia after cerebral revascularization in moyamoya disease. Neurosurgery. 2015;76(3):302-312.
4. Hecht N, Marushima A, Nieminen M, et al. Myoblast-mediated gene therapy improves functional collateralization in chronic cerebral hypoperfusion. Stroke. 2015;46(1):203-211.

5. Hecht N, Peña-Tapia P, Vinci M, et al. Myoblast-mediated gene therapy via encephalomyosynangiosis - a novel strategy for local delivery of gene products to the brain surface. $J$ Neurosci Methods. 2011;201(1):61-66.

6. Hiramatsu M, Hishikawa T, Tokunaga K, et al. Combined gene therapy with vascular endothelial growth factor plus apelin in a chronic cerebral hypoperfusion model in rats. $J$ Neurosurg. 2017;127(3):679-686.

7. Nakamura M, Imai H, Konno K, et al. Experimental investigation of encephalomyosynangiosis using gyrencephalic brain of the miniature pig: histopathological evaluation of dynamic reconstruction of vessels for functional anastomosis. Laboratory investigation. J Neurosurg Pediatr. 2009;3(6):488-495.

8. Ohmori Y, Morioka M, Kaku Y, et al. Granulocyte colonystimulating factor enhances the angiogenetic effect of indirect bypass surgery for chronic cerebral hypoperfusion in a rat model. Neurosurgery. 2011;68(5):1372-1379.

9. Shibata M, Ohtani R, Ihara M, Tomimoto H. White matter lesions and glial activation in a novel mouse model of chronic cerebral hypoperfusion. Stroke. 2004;35(11):2598-2603.

10. Andrae J, Gallini R, Betsholtz C. Role of platelet-derived growth factors in physiology and medicine. Genes Dev. 2008;22(10):1276-1312.

11. Đặng TC, Ishii Y, Nguyen V, et al. Powerful homeostatic control of oligodendroglial lineage by PDGFR $\alpha$ in adult brain. Cell Rep. 2019;27(4):1073-1089.e5.

12. Olson LE, Soriano P. Increased PDGFRalpha activation disrupts connective tissue development and drives systemic fibrosis. Dev Cell. 2009;16(2):303-313.

13. Horikawa S, Ishii Y, Hamashima T, et al. PDGFR $\alpha$ plays a crucial role in connective tissue remodeling. Sci Rep. 2015;5:17948.

14. Lim M, Cheshier S, Steinberg GK. New vessel formation in the central nervous system during tumor growth, vascular malformations, and moyamoya. Curr Neurovasc Res. 2006;3(3):237-245.

15. Kusaka N, Sugiu K, Tokunaga K, et al. Enhanced brain angiogenesis in chronic cerebral hypoperfusion after administration of plasmid human vascular endothelial growth factor in combination with indirect vasoreconstructive surgery. $J$ Neurosurg. 2005;103(5):882-890.

16. Kim HS, Lee HJ, Yeu IS, et al. The neovascularization effect of bone marrow stromal cells in temporal muscle after encephalomyosynangiosis in chronic cerebral ischemic rats. $J$ Korean Neurosurg Soc. 2008;44(4):249-255.

17. Hokari M, Kuroda S, Iwasaki Y. Pretreatment with the ciclosporin derivative NIM811 reduces delayed neuronal death in the hippocampus after transient forebrain ischaemia. $J$ Pharm Pharmacol. 2010;62(4):485-490.

18. Marushima A, Nieminen M, Kremenetskaia I, et al. Balanced single-vector co-delivery of VEGF/PDGF-BB improves functional collateralization in chronic cerebral ischemia. J Cereb Blood Flow Metab. 2020;40(2):404-419.

19. Ulrich PT, Kroppenstedt S, Heimann A, Kempski O. Laser-Doppler scanning of local cerebral blood flow and reserve capacity and testing of motor and memory functions in a chronic 2-vessel occlusion model in rats. Stroke. 1998;29(11):2412-2420.

20. Ignotz RA, Massagué J. Transforming growth factor-beta stimulates the expression of fibronectin and collagen and their incorporation into the extracellular matrix.J Biol Chem. 1986;261(9):4337-4345.

21. Oberringer M, Meins C, Bubel M, Pohlemann T. In vitro wounding: effects of hypoxia and transforming growth factor beta1 on proliferation, migration and myofibroblastic differ- 
entiation in an endothelial cell-fibroblast co-culture model. $J$ Mol Histol. 2008;39(1):37-47.

22. Hurskainen M, Eklund L, Hägg PO, et al. Abnormal maturation of the retinal vasculature in type XVIII collagen/ endostatin deficient mice and changes in retinal glial cells due to lack of collagen types XV and XVIII. FASEB J. 2005;19(11):1564-1566.

23. Sottile J. Regulation of angiogenesis by extracellular matrix. Biochim Biophys Acta. 2004;1654(1):13-22.

24. Aoyagi M, Fukai N, Matsushima Y, et al. Kinetics of 125IPDGF binding and down-regulation of PDGF receptor in arterial smooth muscle cells derived from patients with moyamoya disease. J Cell Physiol. 1993;154(2):281-288.

25. Aoyagi M, Fukai N, Yamamoto M, et al. Development of intimal thickening in superficial temporal arteries in patients with moyamoya disease. Clin Neurol Neurosurg. 1997;99(suppl 2):S213-S217.

26. Kang HS, Kim JH, Phi JH, et al. Plasma matrix metalloproteinases, cytokines and angiogenic factors in moyamoya disease. J Neurol Neurosurg Psychiatry. 2010;81(6):673-678.

27. Schneider CA, Rasband WS, Eliceiri KW. NIH image to Image J: 25 years of image analysis. Nat Methods. 2012;9:671675.

28. Abramoff MD, Magelhaes PJ, Ram SJ. Image processing with ImageJ. Biophotonics Int. 2004;11:36-42.

29. Yamamoto S, Niida S, Azuma E, et al. Inflammation-induced endothelial cell-derived extracellular vesicles modulate the cellular status of pericytes. Sci Rep. 2015;5:8505.

\section{Disclosures}

The authors report no conflict of interest concerning the materials or methods used in this study or the findings specified in this paper.

\section{Author Contributions}

Conception and design: Kuroda. Acquisition of data: Hayashi, Yamamoto, Hamashima, Mori. Analysis and interpretation of data: Kuroda, Hayashi, Yamamoto, Hamashima, Mori. Drafting the article: Kuroda, Hayashi. Critically revising the article: Kuroda, Sasahara. Reviewed submitted version of manuscript: Kuroda, Sasahara. Approved the final version of the manuscript on behalf of all authors: Kuroda. Statistical analysis: Hayashi. Administrative/technical/material support: Kuroda, Sasahara. Study supervision: Kuroda, Sasahara.

\section{Correspondence}

Satoshi Kuroda: Graduate School of Medicine and Pharmaceutical Sciences, University of Toyama, Japan.skuroda@ med.u-toyama.ac.jp. 